Research Article

\title{
Experimental Demonstration of Zadoff-Chu Matrix Transform Precoding for MIMO-OFDM Visible Light Communications
}

\author{
Xinyue Guo, ${ }^{1}$ Chuxian Wang, ${ }^{1}$ and Wei Wang ${ }^{2}{ }^{2}$ \\ ${ }^{1}$ Shanghai Key Lab of Modern Optical System, School of Optical-Electrical and Computing Engineering, \\ University of Shanghai for Science and Technology, Shanghai 200093, China \\ ${ }^{2}$ Naval Medical Center of PLA, Shanghai 200433, China \\ Correspondence should be addressed to Wei Wang; wwang_fd@fudan.edu.cn
}

Received 30 July 2020; Accepted 11 September 2020; Published 21 September 2020

Academic Editor: Junmin Liu

Copyright ( $\odot 2020$ Xinyue Guo et al. This is an open access article distributed under the Creative Commons Attribution License, which permits unrestricted use, distribution, and reproduction in any medium, provided the original work is properly cited.

Visible light communication (VLC) based on a light-emitting diode (LED) is considered to be a potential candidate for the nextgeneration communication. In this paper, a novel Zadoff-Chu matrix transform (ZCT) precoding is proposed to improve the performance of the traditional space-time block coding- (STBC-) based multiple-input multiple-output and orthogonal frequency division multiplexing (MIMO-OFDM) system. Compared with the existing orthogonal circulant matrix transform (OCT) precoding scheme, the proposed ZCT precoding achieves a much lower peak-to-average power ratio (PAPR) while maintaining the advantage of the uniform signal-to-noise ratio (SNR), which reduces the performance loss caused by LED nonlinearity. To study the system performance further, we set up an experimental demonstration to verify performance improvement under the condition of different driving peak-to-peak voltages (Vpps) and direct current (DC) offsets. Experimental results show that ZCT precoding gains the best bit error rate (BER) performance compared with the traditional and the OCT precoding MIMO-OFDM systems, whose BER is always below the $7 \%$ pre-forward error correction (pre-FEC) threshold of $3.8 \times 10^{-3}$.

\section{Introduction}

With high luminous power, small size, good modulation performance, and low cost, white light-emitting diode (LED) is expected to be the recommended lighting equipment for the next generation $[1,2]$. Meanwhile, visible light communication (VLC) has drawn increasing attention in both academia and industry, which supports illumination and communication simultaneously by modulating signals onto the LED [3,4]. Compared with traditional radio frequency (RF) communications, VLC takes the advantages of high efficiency, licensing free, no electromagnetic interference, and high security [5]. Thus, it has been considered as one of the most compelling technologies for supplementing RF communication [6].

One of the most common applications for VLC is the last mile wireless service delivery for the indoor environment. However, the data rate is limited by the modulation bandwidth of the LED [7]. Due to the power-bandwidth tradeoff of LEDs and various parasitic impedances in the LED packaging, signals modulated at high frequencies are strongly attenuated [8]. As a result, many efforts are made to increase the data rate, among which multiple-input multiple-output (MIMO) [9] and orthogonal frequency division multiplexing (OFDM) [10] techniques have been proposed to be combined to form a MIMO-OFDM system to boost capacity and improve reliability $[11,12]$. By dividing the channel into subchannels, OFDM can overcome the intersymbol interference (ISI) and improve spectral efficiency greatly, while MIMO increases the data rate or improves reliability by equipping multiple transmitters and receivers simultaneously [13]. Commonly, multiple LEDs are required to meet the demand for sufficient illumination; thus, it is natural to use the MIMO technique in VLC systems. Since the channel matrix correlation of MIMO VLC systems is usually very high [14], the MIMO scheme based on spacetime block coding (STBC) is considered more suitable for VLC systems than spatial multiplexing, which has been 
proved to be robust to the channel correlation [15]. However, the bit error rate (BER) performance is improved at the cost of sacrificing multiplexing gains in STBC-based MIMO VLC systems.

To achieve a better solution, adaptive modulation design for STBC based on the MIMO-OFDM VLC system was studied in [16], where experimental results confirmed that data rates could be increased greatly without BER performance loss. However, channel state information (CSI) is required to be fed back from the receiver to the transmitter, leading to an additional system overhead [17]. Recently, an orthogonal circulant matrix transform (OCT) was proposed to avoid the feedback, by which uniform signal-to-noise ratio (SNR) could be achieved despite of the nonflat frequency response $[18,19]$. In this way, a similar BER performance is achieved compared with the traditional adaptive modulation scheme, but the feedback overhead can be reduced a lot. However, the peak-to-average power ratio (PAPR) is high in such an OCT precoding MIMO-OFDM system. As the nonlinearity exists when electrical signals are converted into optical signals by the LED, the high PAPR becomes the main factor restricting the system performance [20].

In this paper, we propose and experimentally demonstrate a novel Zadoff-Chu matrix transform (ZCT) [21] precoding scheme for STBC-based MIMO-OFDM VLC systems. By precoding with the Zadoff-Chu (ZC) matrix, the correlation of the input sequence can be reduced, which leads significantly to PAPR suppression. Meanwhile, precoding spreads the information on each subchannel across all the subchannels to achieve the frequency diversity. Thus, the advantage of uniform SNR can still be maintained. The theoretical simulation results show that the PAPR of the ZCT precoding scheme is remarkably lower than that of the traditional STBC and the OCT precoding-based STBC schemes, which is measured in terms of the complementary cumulative distribution function (CCDF). Furthermore, an experimental demonstration is set up to verify the performance improvement, where the system performance is evaluated under the conditions of different driving peak-topeak voltages (Vpps) and direct current (DC) offsets. Experimental results show that the proposed system achieves the best BER performance, whose BER is always below the $7 \%$ pre-forward error correction (pre-FEC) threshold of $3.8 \times 10^{-3}$.

\section{Principle}

2.1. The Proposed MIMO-OFDM VLC System Based on ZCT Precoding. In this section, a novel ZCT precoding-based MIMO-OFDM VLC system is proposed, where STBC is used for its robustness to the channel correlation. Compared with the existing OCT precoding scheme, ZCT precoding maintains the advantage of the ability of SNR equalization but achieves a much lower PAPR. As a result, the performance loss because of the LED nonlinearity can be greatly reduced.

In Figure 1, the block diagram of the proposed MIMOOFDM VLC system with ZCT precoding is illustrated, where the MIMO structure with two transmitters and two receivers is assumed without loss of generality. At the transmitter (TX), original random binary streams are generated; after serialparallel (S/P) conversion and quadrature amplitude modulation (QAM) constellation mapping, ZCT precoding is performed. Then, the precoded signals are encoded by the Alamouti STBC scheme. The training sequences are inserted for channel estimation at the receiver. After the upsampling operation, OFDM modulation is realized by the inverse Fourier transform (IFFT). It should be pointed out that the modulated signals in the frequency domain must be Hermitian symmetrically to ensure that the OFDM signals are real-valued in the time domain. Finally, the cyclic prefix (CP) is added to the OFDM symbol to overcome ISI, and the transmitted signals are generated after parallel-to-serial $(\mathrm{P} / \mathrm{S})$ conversion. At the receiver $(\mathrm{RX})$, inverse processing operations can recover the original data streams. After $\mathrm{S} / \mathrm{P}$ conversion, $\mathrm{CP}$ removing, downsampling, and Fourier transform (FFT) operations, the time-domain signals are transformed to the frequency domain again. Then, with the help of the training sequence, the channel is estimated in the frequency domain. Afterwards, STBC decoding is implemented to remove the effect of the channel. Finally, ZCT decoding and QAM demapping are performed to recover the original binary data streams.

2.2. The Principle of ZCT Precoding. The ZC matrix is generated by the $\mathrm{ZC}$ sequence. The $\mathrm{ZC}$ sequences are a class of polyphase sequences having constant magnitude, ideal periodic autocorrelation, and low cross-correlation properties. In addition, the ZC sequence is still a $\mathrm{ZC}$ sequence after FFT or IFFT. According to Guo et al. [21], the elements of the $\mathrm{ZC}$ sequence can be defined as

$$
z(k)= \begin{cases}e^{(j 2 r \pi / N)\left(\left(k^{2} / 2\right)+q k\right)}, & \text { for } N \text { even, } \\ e^{(j 2 r \pi / N)((k(k+1) / 2) q k)}, & \text { for } N \text { odd },\end{cases}
$$

where $k=0,1,2, \ldots, N-1, q$ is an integer, $N$ is the sequence length, $r$ is an integer relatively prime to $N$, and $j=\sqrt{-1}$.

Then, the ZC matrix can be obtained by transforming the ZC sequence into a square matrix, which is given by

$$
\mathbf{F}=\left[\begin{array}{cccc}
f(0,0) & f(0,1) & \cdots & f(0, M-1) \\
f(1,0) & f(1,1) & \cdots & f(1, M-1) \\
\vdots & \vdots & \ddots & \vdots \\
f(M-1,0) & f(M-1,1) & \cdots & f(M-1, M-1)
\end{array}\right]
$$

In equation (2), the relationship between $f(m, l)$ and $z(k)$ is $f(m, l)=z(m \times M+l)$, where $m$ and $l$ denote the row and column index of the $\mathrm{ZC}$ matrix, respectively. Obviously, the length of the $\mathrm{ZC}$ sequence is equal to the square of the matrix dimension. The normalization factor $1 / \sqrt{M}$ is multiplied by the matrix to normalize the transmitted power. Since the ZC matrix $\mathbf{F}$ is an orthogonal matrix, that is, $\mathbf{F}^{\mathrm{H}} \mathbf{F}=\mathbf{I}$, the inverse matrix of $\mathbf{F}$ always exists and is equal to its conjugate transpose $\mathbf{F}^{\mathrm{H}}$. It is worth noting that there is always a column with the same elements in the $\mathrm{ZC}$ 

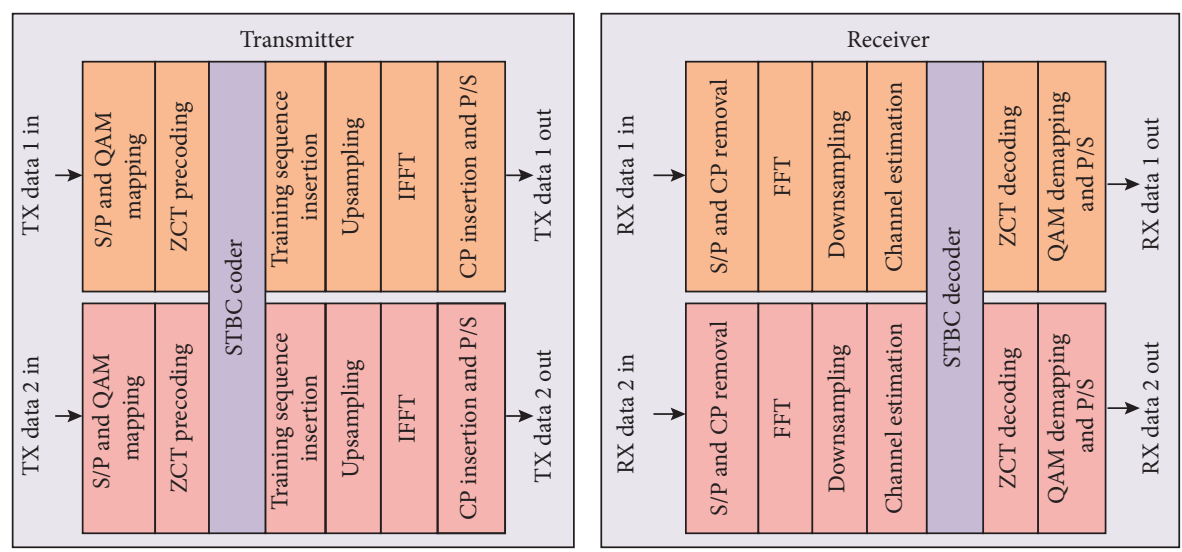

FIGURE 1: Block diagram of the proposed MIMO-OFDM VLC system with ZCT precoding.

matrix, the index of which depends on the value of $q$ and $r$ when the sequence length $N$ is given.

In order to study the performance of ZCT precoding, we establish the mathematical model of ZCT precoding, which is denoted as

$$
\begin{aligned}
& \mathbf{S}_{1}=\mathbf{F} \cdot \mathbf{X}_{1}, \\
& \mathbf{S}_{2}=\mathbf{F} \cdot \mathbf{X}_{2},
\end{aligned}
$$

where $\quad \mathbf{X}_{1}=\left[\begin{array}{llll}x_{1,0} & x_{1,1} & \ldots & x_{1, M-1}\end{array}\right]^{T}$ and $\mathbf{X}_{2}=\left[x_{2,0}\right.$ $\left.x_{2,1} \ldots x_{2, M-1}\right]^{T}$ stand for the data streams after S/P conversion and QAM modulation, $M$ is the number of effective subcarriers carrying the information, and $\mathbf{S}_{1}$ and $\mathbf{S}_{2}$ represent the data streams after precoding.

After OFDM, the time-domain transmitted signals are denoted by

$$
w_{i, p}=\frac{1}{\sqrt{M}} \sum_{m=0}^{M-1} y_{i, m} e^{j(2 \pi m p / M)}, \quad p=0,1, \ldots, M-1,
$$

where $i$ is the transmitter index, $m$ denotes the subcarrier number, and $y_{i, m}$ represents the signal after STBC.

The PAPR of the OFDM signals can be expressed as

$$
\text { PAPR }=\frac{\max \left[\left|w_{i, p}\right|^{2}\right]}{E\left[\left|w_{i, p}\right|^{2}\right]},
$$

where $E[\cdot]$ represents the expectation operation.

As can be seen, signals on each subchannel are multiplied by a phase rotation factor and then summed up. Thus, the phases and energies are reallocated through precoding. This leads to the reduction of the correlation of the input sequences, and finally, the PAPR can be greatly suppressed.

At the receiver, the estimated signals after ZCT decoding can be expressed as

$$
\begin{aligned}
& \widehat{\mathbf{X}}_{1}=\mathbf{F}^{H} \widehat{\mathbf{S}}_{1}, \\
& \widehat{\mathbf{X}}_{2}=\mathbf{F}^{\mathrm{H}} \widehat{\mathbf{S}}_{2},
\end{aligned}
$$

where $\quad \widehat{\mathbf{S}}_{1}=\left[\begin{array}{llll}\widehat{\boldsymbol{s}}_{1,0} & \widehat{\boldsymbol{s}}_{1,1} & \ldots & \widehat{\boldsymbol{s}}_{1, M-1}\end{array}\right]^{T}$ and $\quad \widehat{\mathbf{S}}_{2}=\left[\begin{array}{ll}\widehat{\boldsymbol{s}}_{2,0} & \end{array}\right.$ $\left.\widehat{s}_{2,1} \ldots \widehat{s}_{2, M-1}\right]^{T}$ are the signals after STBC decoding.

So, the SNR of the $m^{\text {th }}$ subchannel for the $i^{\text {th }}$ transmitter is calculated by

$$
\mathrm{SNR}_{i, m}=\frac{1}{N_{0} \mathbf{F}_{m}^{H}\left[\left(\left\|\mathbf{H}_{0}\right\|_{F}^{2}\right)^{-1}\left(\left\|\mathbf{H}_{1}\right\|_{F}^{2}\right)^{-1} \ldots\left(\left\|\mathbf{H}_{M-1}\right\|_{F}^{2}\right)^{-1}\right]^{T}}
$$

where the power of transmitted signals is assumed normalized, $N_{0}$ represents the noise power, $\|\cdot\|_{F}$ denotes the Frobenius 2-norm, $\mathbf{H}_{m}$ is the channel matrix of the $m^{\text {th }}$ subchannel, and $\mathbf{F}_{m}^{H}$ is the $m^{\text {th }}$ row of $\mathbf{F}^{\mathrm{H}}$. Equation (7) indicates that SNRs are uniform in the subchannels except in one subchannel. Because of ZCT precoding, the information on each subchannel is spread across all the subchannels. Therefore, frequency diversity is achieved for most of the subchannels. However, as mentioned above, there is one column with the same elements in the ZC matrix. Obviously, frequency diversity cannot be achieved for the subchannel corresponding to the special column. As a result, the subchannel should be avoided in preventing performance loss.

\section{Experiment Setup and Results}

In this section, we set up an experimental demonstration to study the system performance further. The experimental setup of the $2 \times 2$ MIMO-OFDM VLC system is shown in Figure 2. At the transmitter, the generated signals are loaded into an arbitrary function generator (AFG: Tektronix AFG3252C). DC offset supplied by the AFG is also added to ensure the signals are positive. Then, mixed signals are transmitted by two commercially available LEDs (Cree XLamp XP-E: the center wavelength is $620 \mathrm{~nm}$, and the maximum power is $1 \mathrm{~W}$ ) in the form of the optical power. As shown in Figure 2, the LED is a point light source radiating red light; in order to make use of the optical power sufficiently, a reflection cup with $60^{\circ}$ is used to concentrate the light. At the receiver, the optical signals are converted into electrical signals by two photodetectors (PDs) (Hamamatsu $\mathrm{C} 12702-11,0.42 \mathrm{~A} / \mathrm{W}$ responsivity at $620 \mathrm{~nm}$ ). After the electrical signals are amplified by two electrical amplifiers 


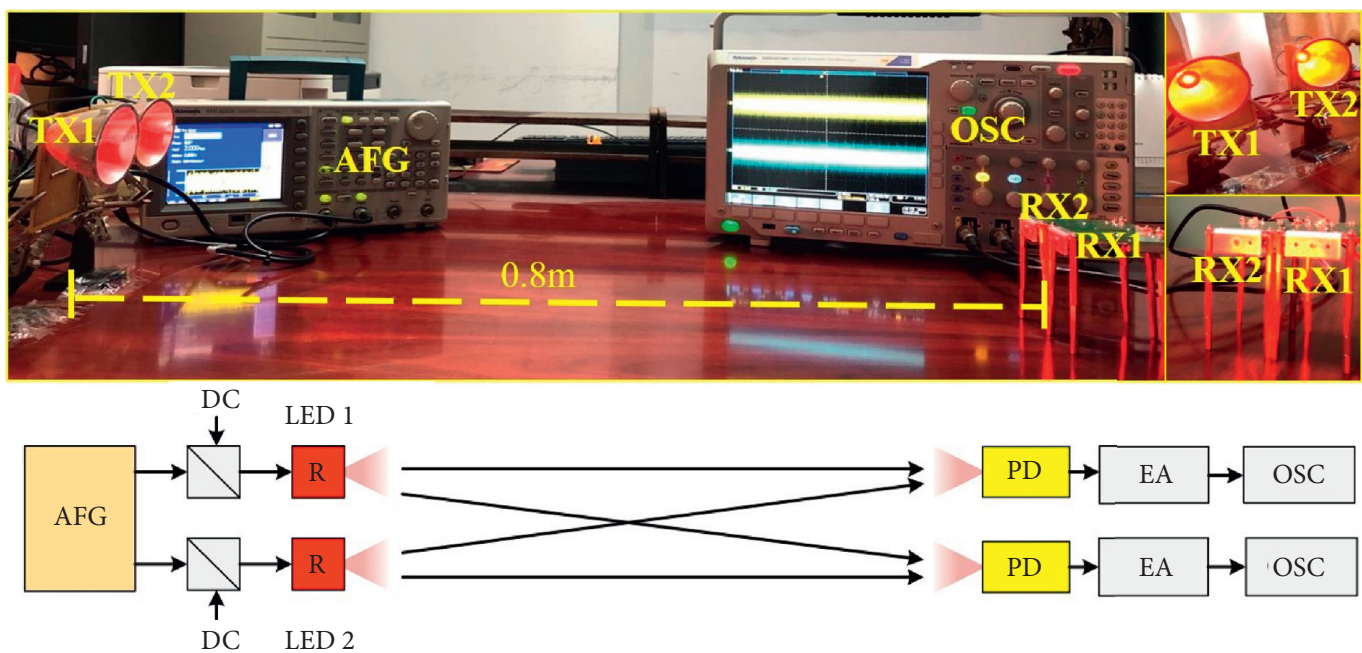

Figure 2: Experimental setup of the MIMO-OFDM VLC system based on ZCT precoding.

(EAs), the received signals are recorded by a high-speed digital oscilloscope (OSC: Tektronix MDO4104C). Finally, the recorded signals are sent for offline processing.

The parameters used in the experiment are listed in Table 1, where 64QAM and 128QAM modulation formats are chosen for the two transmitters, respectively, to achieve the data rate as high as possible under the BER constraint of the $7 \%$ pre-FEC threshold of $3.8 \times 10^{-3}$. Considering the poor frequency response of the first few subcarriers, zeropadding is used for the first six subcarriers. As a result, only 122 subcarriers are available for data transmission. In the experiment, the same ZC matrix is chosen for the two data streams for simplicity, where parameters $q$ and $r$ of the ZC sequence are set as 30 and 1, respectively. In this case, the elements of the $32^{\text {nd }}$ column in the ZC matrix are identical as discussed above, and the $32^{\text {nd }}$ subcarrier is not utilized for data transmission. Therefore, with an available bandwidth of $\sim 12.5 \mathrm{MHz}$, the proposed system can realize the data rate of $\sim 38.4 \mathrm{Mb} / \mathrm{s}$.

Firstly, PAPR is measured by the complementary cumulative distribution function (CCDF), which denotes the probability that the PAPR of the OFDM symbols exceeds a given threshold $\mathrm{PAPR}_{0}$ [22]. The statistics of the PAPR of OFDM signals can be expressed as

$$
\mathrm{CCDF}=\operatorname{Prob}\left(\mathrm{PAPR}>\mathrm{PAPR}_{0}\right) .
$$

Figure 3illustrates the simulation results of CCDF curves for ZCT precoding, OCT precoding, and traditional MIMOOFDM schemes. As shown in Figure 3, the PAPR performance is similar for the OCT precoding and traditional systems, while the PAPR of the ZCT precoding-based MIMO-OFDM system is much lower. At the clip rate of $10^{-4}$, there is about more than $2 \mathrm{~dB}$ gain compared to the PAPR of the proposed scheme with the other two schemes.

Then, the SNRs of subchannels are measured in different systems as shown in Figure 4, where Figures 4(a) and 4(b) represent the SNR profiles of RX1 and RX2, respectively. The results confirm that the SNR can be equalized to the identical level in the two MIMO-OFDM systems with precoding, but
TABLE 1: Experiment parameters.

\begin{tabular}{lc}
\hline Parameters & Values \\
\hline Modulation order & 64QAM, 128QAM \\
Bandwidth & $12.5 \mathrm{MHz}$ \\
Subcarrier number & 256 \\
CP length & 16 \\
Upsampling rate & 4 \\
Transmission distance & $0.8 \mathrm{~m}$ \\
Distance between TXs & $5 \mathrm{~cm}$ \\
Distance between RXs & $2 \mathrm{~cm}$ \\
\hline
\end{tabular}

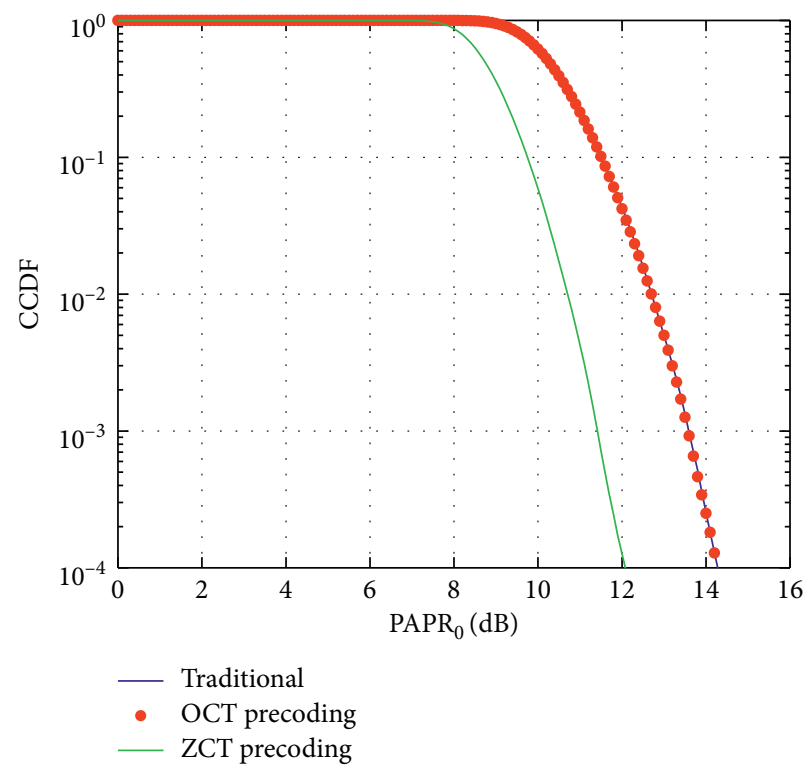

FIgure 3: CCDF of PAPRs for different MIMO-OFDM VLC schemes.

the SNR of the traditional MIMO-OFDM system is severely attenuated with the frequency increasing. Note that the $32^{\text {nd }}$ subcarrier is not used in the ZCT precoding-based system, so the SNR curve is discontinuous in that subcarrier. 


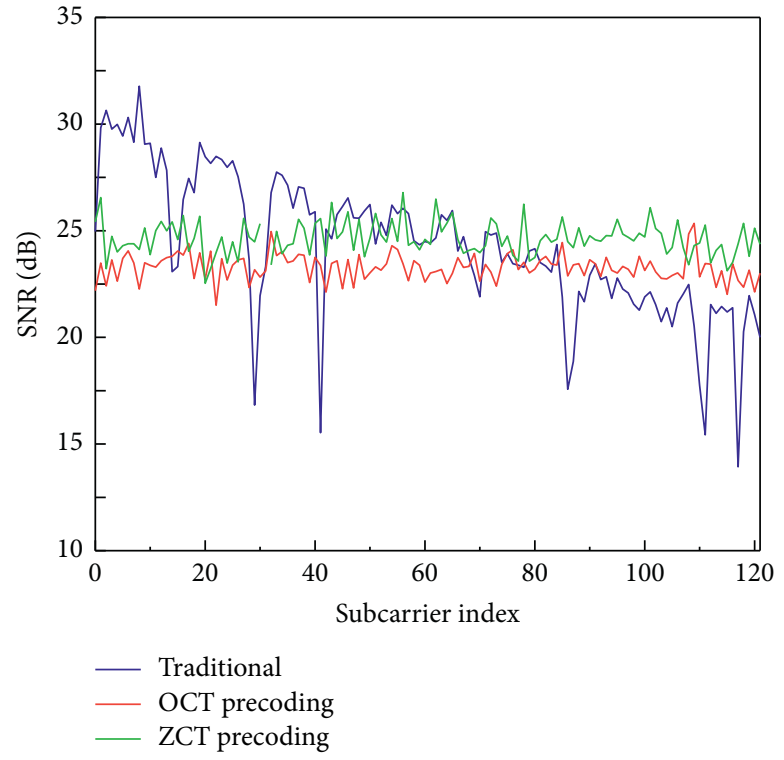

(a)

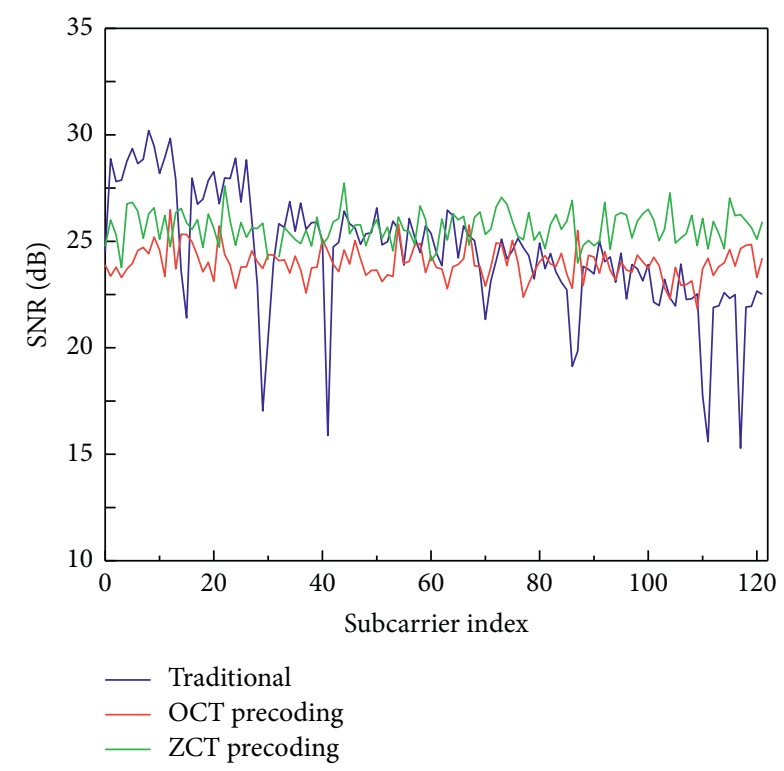

(b)

FIgURE 4: The SNR profiles of different MIMO-OFDM VLC schemes (DC=2 V, Vpp=2 V): (a) the SNR profiles of RX1; (b) the SNR profiles of RX2.

As discussed above, high PAPR has a great impact on the system performance as the nonlinearity exists during the electro-optical conversion by the LED. In Figure 5, the relationship between the driving voltage and the forward current of the LED we used is represented. It can be seen that the linear region of the U-I curve is located when the voltage is between $1.9 \mathrm{~V}$ and $2.2 \mathrm{~V}$. When the voltage is below $1.9 \mathrm{~V}$ or above $2.2 \mathrm{~V}$, hard clipping or nonlinear mapping would occur, which causes nonlinear distortion of the transmitted signals [23]. As well known, the higher the PAPR is, the greater the probability of nonlinear distortion would be. Therefore, the system with higher PAPR suffers from more serious BER performance loss.

Furthermore, BER is measured to study the impact of the PAPR on the system performance. In Figure 6, the BERs of the three systems under the condition of different DC offsets are compared, where the DC offsets are set from $1.8 \mathrm{~V}$ to $2.5 \mathrm{~V}$. In the experiment, the $\mathrm{Vpp}$ is fixed as $2 \mathrm{~V}$. With the DC offset increasing, for each curve, it can be seen that the BER decreases initially and then increases. The best BER performance is obtained when the DC offset is set as $2.1 \mathrm{~V}$. This result is reasonable since $2.1 \mathrm{~V}$ is exactly located in the middle of the linear voltage region of the LED. Compared with the three schemes, the ZCT precoding scheme performs best, whose BERs are always below the $7 \%$ pre-FEC threshold of $3.8 \times 10^{-3}$. Experimental results validate the theoretical analysis that ZCT precoding outperforms OCT precoding due to its superiority in the PAPR. Meanwhile, the uniform feature of the SNR also improves the performance, which can be proved by the result comparison of the OCT precoding and the traditional schemes. Benefiting from the effect of the SNR equalization, the errors caused by high-

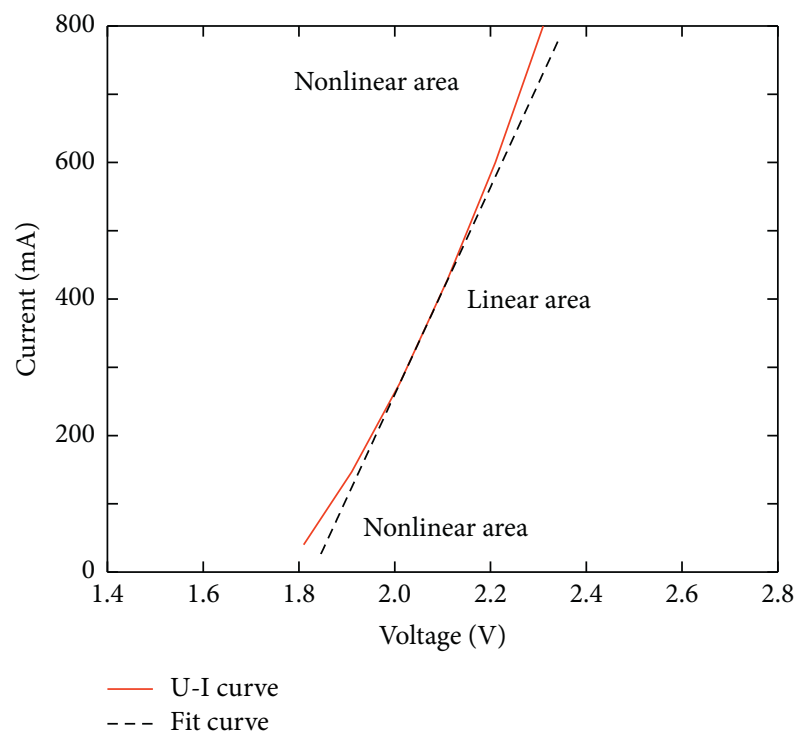

FIGURE 5: U-I curve of the LED.

frequency attenuation are greatly reduced, and the BER performance can be improved.

Moreover, the BER performance of different schemes is also evaluated by changing the $\mathrm{Vpp}$ values from $1.6 \mathrm{~V}$ to $2.8 \mathrm{~V}$ when the DC offset is set equal to $2.1 \mathrm{~V}$, as depicted in Figure 7. The experimental results show that the BER decreases with the Vpp increasing at first. This is because the SNR can be enhanced by increasing the Vpp. However, BER increases again, indicating that nonlinear distortion plays the dominant role when the Vpp keeps increasing. Nevertheless, the ZCT precoding scheme still achieves the best performance compared with the other two schemes. 


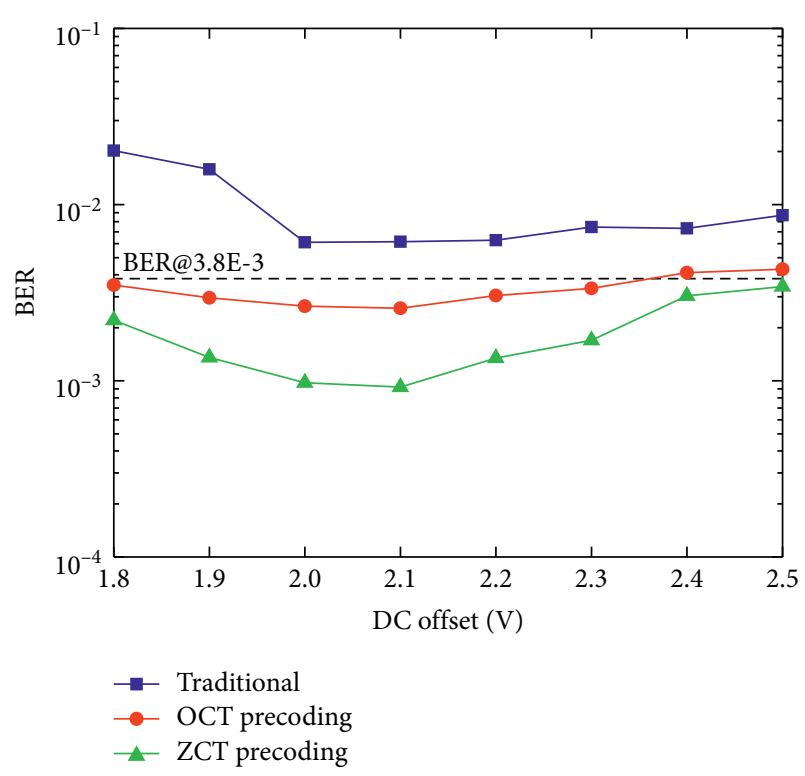

Figure 6: BER versus DC offset $(\mathrm{Vpp}=2 \mathrm{~V})$.

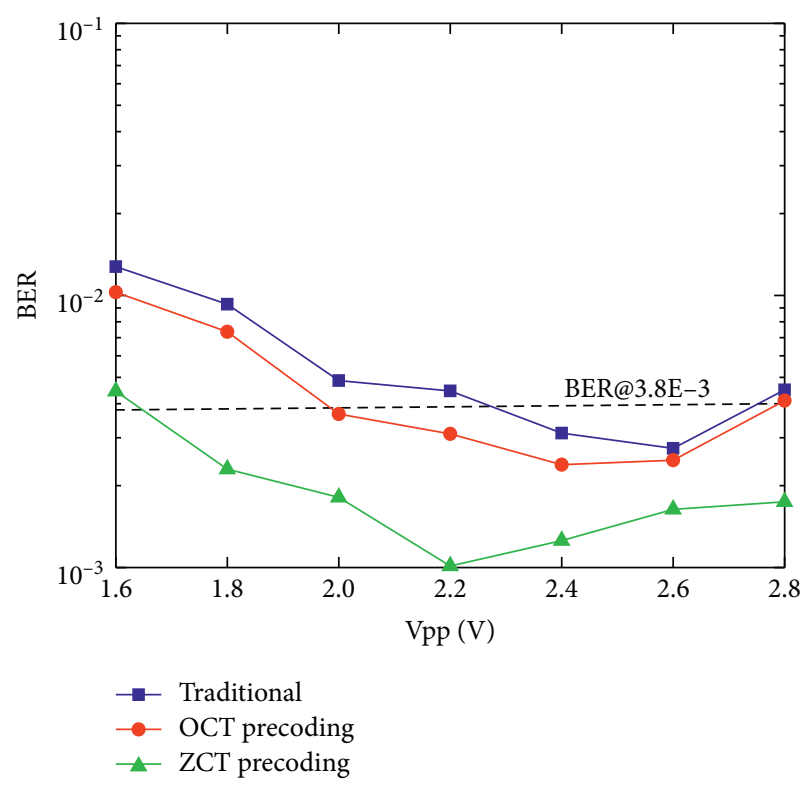

Figure 7: BER versus Vpp $(\mathrm{DC}=2.1 \mathrm{~V})$.

\section{Conclusion}

In this paper, we propose and experimentally demonstrate a novel MIMO-OFDM VLC system based on ZCT precoding, where STBC is chosen as the MIMO mechanism to cope with the high channel correlation. Considering the frequency attenuation characteristics and the nonlinear U-I curve of the LED, ZCT precoding is able to obtain the uniform SNR based on the idea of frequency diversity to reduce the performance loss of the frequency attenuation. At the same time, a much lower PAPR is also achieved by reallocating the phases and energies among the subchannels so as to avoid the nonlinear distortion caused by the LED as much as possible. Detailed explorations of the proposed system performance are experimentally investigated by changing the driving Vpps and DC offsets. Experimental results indicate that the best BER performance can be achieved by the proposed ZCT precoding MIMO-OFDM system compared with the traditional and the OCT precoding system, where the BER can always be below the $7 \%$ pre-FEC threshold of $3.8 \times 10^{-3}$.

\section{Data Availability}

All the data used to support the findings of this study are available from the corresponding author upon request.

\section{Conflicts of Interest}

The authors declare that they have no conflicts of interest.

\section{Acknowledgments}

This work was supported by the Foundation of China (no. HJ20172B0202718K503).

\section{References}

[1] N. Chi, H. Haas, M. Kavehrad, T. D. C. Little, and X.-L. Huang, "Visible light communications: Demand factors, benefits and opportunities [Guest Editorial]," IEEE Wireless Communications, vol. 22, no. 2, pp. 5-7, 2015.

[2] H. Marshoud, S. Muhaidat, P. C. Sofotasios, S. Hussain, M. A. Imran, and B. S. Sharif, "Optical non-orthogonal multiple access for visible light communication," IEEE Wireless Communications, vol. 25, no. 2, pp. 82-88, 2018.

[3] J.-Y. Wang, Q.-L. Li, J.-X. Zhu, and Y. Wang, "Impact of receiver's tilted angle on channel capacity in VLCs," Electronics Letters, vol. 53, no. 6, pp. 421-423, 2017.

[4] A. M. Vegni and M. Biagi, "Optimal LED placement in indoor VLC networks," Optics Express, vol. 27, no. 6, pp. 8504-8519, 2019.

[5] X. Huang, Z. Wang, J. Shi, Y. Wang, and N. Chi, "16 Gbit/s phosphorescent white LED based VLC transmission using a cascaded pre-equalization circuit and a differential outputs PIN receiver," Optics Express, vol. 23, no. 17, pp. 22034-22042, 2015.

[6] P. H. Pathak, X. Feng, P. Hu, and P. Mohapatra, "Visible light communication, networking, and sensing: A survey, potential and challenges," IEEE Communications Surveys \& Tutorials, vol. 17, no. 4, pp. 2047-2077, 2015.

[7] P. Deng, M. Kavehrad, and M. A. Kashani, "Nonlinear modulation characteristics of white LEDs in visible light communications," in Proceedings of the Optical Fiber Communications Conference and Exhibition, pp. 1-3, Los Angeles, CA, USA, 2015.

[8] A. Jovicic, J. Li, and T. Richardson, "Visible light communication: Opportunities, challenges and the path to market," IEEE Communications Magazine, vol. 51, no. 12, pp. 26-32, 2013.

[9] W. Wang and W. Zhang, "Signal shaping and precoding for MIMO systems using lattice codes," IEEE Transactions on Wireless Communications, vol. 15, no. 7, pp. 4625-4634, 2019.

[10] Q. Hu, X. Jin, and Z. Xu, "Compensation of sampling frequency offset with digital interpolation for OFDM-based visible light communication systems," Journal of Lightwave Technology, vol. 36, no. 23, pp. 5488-5497, 2018. 
[11] L. Deng and Y. Fan, "Analysis of channel correlation and channel capacity for indoor MIMO visible light communication systems," Applied Optics, vol. 59, no. 15, pp. 4672-4684, 2020.

[12] X. Yan, J. He, Y. Liu et al., "A polar-coded MIMI-OFDM scheme for VLC system," in Proceedings of the Asia Communications and Photonics Conference and Exhibition, Chengdu, China, November 2019.

[13] A. Kumar and S. K. Ghorai, "BER performance analysis of indoor MIMO-VLC system for multipath reflection," in Proceedings of the 2018 Technologies for Smart-City Energy Security and Power, pp. 1-5, Bhubaneswar, India, March 2018.

[14] M. L. Tran and S. Kim, "Orientation-induced link-blocked receiver for MIMO visible light communication," Optics Express, vol. 28, no. 8, p. 12157, 2020.

[15] C.-C. Wei, F.-M. Wu, Z.-Y. Chen et al., "Indoor VLC system with multiple LEDs of different path lengths employing spacetime block-coded DMT/CAP modulation [invited]," Journal of Optical Communications and Networking, vol. 7, no. 3, pp. A459-A466, 2015.

[16] X. Guo, S. Li, Y. Guo et al., "Design of an adaptive STBC MIMO-OFDM system for indoor visible light communication," Infrared and Laser Engineering, vol. 47, no. 2, pp. 191-198, 2018.

[17] Y. Hong, T. Wu, and L.-K. Chen, "On the performance of adaptive MIMO-OFDM indoor visible light communications," IEEE Photonics Technology Letters, vol. 28, no. 8, pp. 907-910, 2016.

[18] Y. Hong, L.-K. Chen, and J. Zhao, "Performance-enhanced gigabit/s MIMO-OFDM visible light communications using CSI-free/dependent precoding techniques," Optics Express, vol. 27, no. 9, pp. 12806-12816, 2019.

[19] Y. Hong, J. Xu, and L.-K. Chen, "Experimental investigation of multi-band OCT precoding for OFDM-based visible light communications," Optics Express, vol. 25, no. 11, pp. 12908-12914, 2017.

[20] H. Lu, Y. Hong, L. K. Chen, and J. Wang, "On the study of the relation between linear/nonlinear PAPR reduction and transmission performance for OFDM-based VLC system," Optics Express, vol. 26, no. 11, 2018.

[21] X. Guo, S. Li, and Y. Guo, "Experimental demonstration of special-shaped 32-quadrature amplitude modulation constellations for visible light communications," Advances in Condensed Matter Physics, vol. 2018, pp. 1-7, 2018.

[22] C. Geetha Priya and M. Suganthi, "Combined PAPR reduction and frequency offset estimation using precoded ZadoffChu OFDM in WLAN system," in Proceedings of the 3rd IEEE International Conference on Computer Science and Information Technology, pp. 461-465, Chengdu, China, July 2010.

[23] J. Zhao, C. Qin, M. Zhang, and N. Chi, "Investigation on performance of special-shaped 8-quadrature amplitude modulation constellations applied in visible light communication," Photonics Research, vol. 4, no. 6, pp. 249-256, 2016. 